OPEN ACCESS

Edited by:

Alfredo Pulvirenti,

University of Catania, Italy

Reviewed by:

Nitish Kumar Mishra,

University of Nebraska Medical

Center, United States

Vsevolod Jurievich Makeev,

Russian Academy of Sciences,

Russia

*Correspondence:

Yanying Zhang

15687386@qq.com

Kehu Yang

kehuyangebm2006@126.com

tThese authors have contributed equally to this work

Specialty section:

This article was submitted to

Bioinformatics and Computational

Biology,

a section of the journal

Frontiers in Genetics

Received: 06 April 2019 Accepted: 05 September 2019

Published: 11 October 2019

Citation:

Bing Z, Yao Y, Xiong J, Tian J, Guo $X$, Li X, Zhang J, Shi X, Zhang Y and Yang K (2019) Novel Model for Comprehensive Assessment of Robust Prognostic Gene Signature in Ovarian Cancer Across Different Independent Datasets.

Front. Genet. 10:931.

doi: 10.3389/fgene.2019.00931

\section{Novel Model for Comprehensive Assessment of Robust Prognostic Gene Signature in Ovarian Cancer Across Different Independent Datasets}

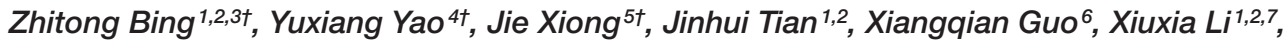 \\ Jingyun Zhang ${ }^{1,2}$, Xiue Shi ${ }^{8}$, Yanying Zhang ${ }^{9 *}$ and Kehu Yang ${ }^{1,2,8,9 *}$ \\ ${ }^{1}$ Evidence Based Medicine Center, School of Basic Medical Science of Lanzhou University, Lanzhou, China, ${ }^{2}$ Key Laboratory \\ of Evidence Based Medicine and Knowledge Translation of Gansu Province, Lanzhou, China, ${ }^{3}$ Department of Computational \\ Physics, Institute of Modern Physics, Chinese Academy of Sciences, Lanzhou, China, ${ }^{4}$ School of Physical Science and \\ Technology, Lanzhou University, Lanzhou, China, ${ }^{5}$ Department of Applied Mathematics, Changsha University, Changsha, \\ China, ${ }^{6}$ Medical Bioinformatics Institute, School of Basic Medicine, Henan University, Henan, China, ${ }^{7}$ School of Public \\ Health, Lanzhou University, Lanzhou, China, ${ }^{8}$ Institute for Evidence Based Rehabilitation Medicine of Gansu Province, \\ Lanzhou, China, ${ }^{9}$ Department of Pharmacology and Toxicology of Traditional Chinese Medicine, Gansu University of Chinese \\ Medicine, Lanzhou, China
}

Different analytical methods or models can often find completely different prognostic biomarkers for the same cancer. In the study of prognostic molecular biomarkers of ovarian cancer (OvCa), different studies have reported a variety of prognostic gene signatures. In the current study, based on geometric concepts, the linearity-clustering phase diagram with integrated P-value (LCP) method was used to comprehensively consider three indicators that are commonly employed to estimate the quality of a prognostic gene signature model. The three indicators, namely, concordance index, area under the curve, and level of the hazard ratio were determined via calculation of the prognostic index of various gene signatures from different datasets. As evaluation objects, we selected 13 gene signature models (Cox regression model) and 16 OvCa genomic datasets (including gene expression information and follow-up data) from published studies. The results of LCP showed that three models were universal and better than other models. In addition, combining the three models into one model showed the best performance in all datasets by LCP calculation. The combination gene signature model provides a more reliable model and could be validated in various datasets of OvCa. Thus, our method and findings can provide more accurate prognostic biomarkers and effective reference for the precise clinical treatment of OvCa.

Keywords: ovarian cancer, prognosis index, Cox regression, gene signature, robust prognostic model

\section{INTRODUCTION}

Ovarian cancer $(\mathrm{OvCa})$ is the most prevalent lethal gynecological malignancies and the fifth-leading cause of cancer death among the female population in the United States, with 21,880 new cases and 13,850 deaths in 2009 (Jemal et al., 2010). In 2018, 22,530 new cases and 13,980 deaths were reported in USA (Siegel et al., 2019). Based on the above statistical data and the results from publications 
on research on OvCa, we found that during nearly 10 years, the incidence and mortality of OvCa have not significantly decreased. The main reason for this is generally considered to be the clinically heterogeneous nature of OvCa. Patients with morphologically similar, advanced-stage tumor display a broad range of clinical outcomes. Prognostic factors, including age, performance status, tumor node metastasis (TNM) stage, histological grade and subtype, and initial surgery results, are insufficient to reflect the important individual variation in response to chemotherapy and to assess survival time among various therapeutic strategies.

The aim of this study is to find a method to evaluate different prognostic gene signature in the OvCa. We found that different studies have found completely different biomarkers for the same cancer. In this era of personalized medicine, molecular biomarkers as important factors for promoting prognosis are being studied comprehensively. With increasing number of clinical cases and the availability of corresponding gene expression profiles, many studies from 2005 to 2017 have provided gene signatures for predicting prognosis of $\mathrm{OvCa}$ (Bonome et al., 2005; Bonome et al., 2008; Crijns et al., 2009; Denkert et al., 2009; Mok et al., 2009; Hernandez et al., 2010; Konstantinopoulos et al., 2010; Yoshihara et al., 2010; Network TCGA, 2011; Sabatier et al., 2011; Bentink et al., 2012; Gyorffy et al., 2012; Kang et al., 2012; Kernagis et al., 2012; Yoshihara et al., 2012; Verhaak et al., 2013; Riester et al., 2014; Waldron et al., 2014; Willis et al., 2016; Yang et al., 2016; Nguyen et al., 2017). Despite this, the prognosis for OvCa has not improved significantly as expected. In the above studies, various types of biomarkers were obtained by diverse statistical methods, platforms, and patient sample sets, leading to irreproducible results. For multi-platform data, meta-analysis is a very effective way to integrate data from different sources ( $\mathrm{Li}$ et al., 2014; Ge et al., 2018). Hence, some studies extracted novel gene signature by merging datasets and meta-analysis (Riester et al., 2014; Waldron et al., 2014). Additionally, some studies applied other methods to estimate the quality of gene signature in OvCa. For instance, Waldron et al. (2014) estimated the performance of each gene signature model by comparing with the random gene group, whereas Riester et al. (2014) merged different data and provided a novel gene signature model. With the development of powerful statistical methods and the increasing amount of data, new biomarkers for predicting OvCa outcome have been put forward (Willis et al., 2016; Yang et al., 2016; Nguyen et al., 2017).

From the perspective of medical statistics, larger sample size indicates more accuracy and confidence level. Moreover, numerous clinical factors and heterogeneity of population and diseases lead to poor performance of distinction even in the same sample sizes. Even though diverse microarray platforms have made enormous progress in accuracy, variations among platforms cannot be ignored when drawing conclusions associated with key gene expression or gene signatures (Barnes et al., 2005; Crijns et al., 2009; Thomson and Dinger, 2016). Above-mentioned publications of OvCa gene signature also utilized different training or testing sample sizes and platforms, and few researchers focused on both sample practicability and risk gene universality.
Generally, the indicators for assaying the performance of prognostic model are concordance index (C-index), hazard ratio (HR), area under the curve (AUC) of receiver operating characteristic (ROC) curve, and P-value of the logrank test. Although any one of the indicators can estimate the performance of prognostic model, it is not clear how to integrate these indicators for reasonable evaluation of the prognostic model, because each indicator represents different attributes in one model. There is insufficient evidence to show that a single index can effectively prove the stability and universality of a model. Thus, to construct a reasonable integrative evaluation method that we proposed, LCP method is needed. Moreover, when all the indicators are not uniform, it is difficult to judge whether a model is good or bad. For example, it is difficult to estimate the performance of a model with high HR and low AUC level. In the research of systematic evaluation, researchers have developed many tools to evaluate medical guidelines (Norris et al., 2016). However, it still remains difficult to estimate quality of genome research and select an appropriate gene signature model. Thus, we proposed a method to integrate these indicators and directly show the model with better performance. Firstly, prognostic index (PI) as a discrimination index for classifying patients into highrisk and low-risk groups was calculated by linear combination of gene signature expression and their coefficients from Cox regression. Secondly, HR, C-index, AUC, and P-value for PI were computed across different datasets. Thirdly, we mapped the value of $\mathrm{HR}, \mathrm{C}$-index, and AUC into three-dimensional space coordinate system and evaluated the models from geometric property, linearity, and clustering. Additionally, P-value used color depth for indicting distinguishing ability of model.

\section{MATERIALS AND METHODS}

\section{Data Collection and Filtering Process}

In order to ensure coincident statistical protocols, we only focused on models obtained from Cox regression methods. Based on this, we filtered out 13 models with gene signature by Cox regression. For assessing each model, we found $16 \mathrm{OvCa}$ sample sets from two databases, The Cancer Genomic Atlas (TCGA) and Gene Expression Omnibus (GEO). The TCGAOvCa dataset, employed by three platforms (Affymetrix HT U133a, Illumina HiSeq V2, AgilentG450), were considered as three different sub-datasets to assess the influence of platform diversity. From the GEO database, we collected 13 sample sets of OvCa patients from 2005 to 2017, namely, GSE19161 (Konstantinopoulos et al., 2011), GSE3149 (Bild et al., 2006), GSE9899 (Tothill et al., 2008), GSE26712 (Bonome et al., 2008), GSE14764 (Denkert et al., 2009), GSE18520 (Mok et al., 2009), GSE17260 (Yoshihara et al., 2010), GSE26193 (Mateescu et al., 2011), GSE32062 (Yoshihara et al., 2012), GSE30009 (Gillet et al., 2012), GSE63885 (Lisowska et al., 2014), GSE13876 (Crijns et al., 2009), and GSE19829 (Thomson and Dinger, 2016). Only samples satisfying the following three conditions were viewed as valid: (1) complete mRNA expression profile 
and clinical information, (2) survival information on patient, and (3) the tumor was primary tumor.

\section{Preprocessing of mRNA Expression Profiles Associated With Survival Analysis}

The three platforms in TCGA have their own characteristics (value of Affymetrix HT U133a is positive with the magnitude of $10^{0} \sim 10^{1}$, AgilentG450 has both positive and negative values, and Illumina HiSeq V2 included more zero than the other two platforms). Since there was no distinct difference between the absolute value of the three platforms, each platform was considered as an independent sample set, and we retained the original expression matrix to keep platform characteristics. The 13 sample sets in GEO were collected from published literature. Each dataset has its own research topic or purpose, test time and date, and different sample sizes. Some expression profiles are provided by probes not genes, such that one gene may map multiple probes, leading to the "several-for-one" matching phenomena. The expression levels of various probes that map the same gene are approximate; therefore, we integrated multiprobes as one gene by getting their arithmetic average to prevent repeated calculations. The same processing was implemented for multiple probe biomarkers (see below for details).

\section{Obtaining and Processing HR Value}

The 13 collected models provided their own HR or coefficient values $(\beta)$ by means of Cox regression method, and the two types of factor can be transformed by the formula $\beta=\log (\mathrm{HR})$. All gene signature models were utilized by following analysis for calculating PI/NPI in all datasets. For simplicity and consistency, all $H R$ values were transformed into $\beta$. As mentioned above, one gene can be detected by multiple probes, which have different HRs; in such cases, we integrated the probes using mean values.

\section{PI Evaluation of Risk Gene Groups/ Signature}

PI, as an estimate of one patient's risk, is the linear combination of risk coefficient multiplied by corresponding mRNA expression in its standard form, namely, normalized prognostic index (NPI), which can reflect one patient deviation in patient sets. We used the gene signature from each model to construct PI/NPI.

$$
\begin{aligned}
\mathrm{PI} & =\sum_{i}\left(\beta_{i} \times X_{i}\right) \\
\mathrm{NPI} & =\frac{\mathrm{PI}-\operatorname{mean}(\mathrm{PI})}{\mathrm{SD}(\mathrm{PI})}
\end{aligned}
$$

where $\mathrm{X}_{i}$ is the value of the $i$ th variable with its regression coefficient $\beta_{i}$. For PI, $X_{i}$ is the mRNA expression value of each risk gene in each model, and $\beta_{i}$ is the coefficient of Cox regression of the $i$ th gene. After calculating each patient's NPI in one dataset, the median NPI was used as the cutoff point to classify patients into high-risk (with NPI greater than median value) and low-risk groups.

For assessing each gene signature model performance among various datasets, we treated each sample set as an independent one. According to the NPI of each patient, we classified patients into high-/low-risk groups. Then, we analyzed each sample set's overall survival (OS) difference between the two groups based on KaplanMeier survival curves and HR with 95\% confidence interval calculated by univariate Cox regression analysis based on NPI. Especially, the HR obtained here was characterized as one model's $\mathrm{HR}$ and not at gene level, and one gene signature model was verified in one dataset only, resulting in one HR value. P-value of log-rank test (two-sided test) was used to determine the difference between high-risk and low-risk groups. Similarly, we also calculated the AUC of the corresponding ROC curve and obtained the C-index of each model in various datasets. For all above-mentioned data, the filtering, preprocessing, and survival analysis were done using R (V.3.5.1), (Ihaka and Gentleman, 1996), with help of the survival and survivalROC package (Heagerty et al., 2000).

\section{Integrated Assessment of Models From Geometric Approach: LCP Methods}

The main purpose of this study was to evaluate the prognostic ability of various $\mathrm{OvCa}$ risk models in an integrated geometric method. Therefore, using collinearity verification (Supplementary Figure S1), we integrated the indicators, namely, HR, C-index, and AUC as model-in-dataset coordinates. In detail, we considered one model's performance in all 16 datasets as a group of three-dimensional (3D) scatter points in clinical indicator space (all three indexes of $\mathrm{HR}, \mathrm{C}$-index, and AUC as independent coordinates). We transformed the three factors into same interval as follows:

$$
\begin{gathered}
\mathrm{HR}^{\prime}=\log \mathrm{HR} \\
\mathrm{C}_{\text {index }}^{\prime}=\log \left(2 \times \mathrm{C}_{\text {index }}\right) \\
\mathrm{AUC}^{\prime}=\log (2 \times \mathrm{AUC})
\end{gathered}
$$

Thus, trivial values of HR, C-index, and AUC (1.0, 0.5, and 0.5 respectively) would be transferred into zero. For the $3 \mathrm{D}$ scatter points, we proposed two geometric concepts-linearity and clustering-reflecting one model's consistency and robustness, respectively. The residual of the best fitting 3D straight line through all scatter points stands for linearity, and clustering was obtained by $3 \mathrm{D}$ scatter points' first moment and second moment (see the following equations):

$$
\text { Residual }=\sum_{i} \mathrm{DL}(\mathrm{x}, \mathrm{y}, \mathrm{z})_{i}
$$

where DL is a function that calculates the distance between one $3 \mathrm{D}$ point and the best fitting line in one model, and the 
summation notation indicates consideration of each point (the 16 datasets) together to obtain the model's residual; $\mathrm{x}, \mathrm{y}$, and $\mathrm{z}$ are HR,' C-index, and AUC' values of one model in one specified dataset, respectively; $\vec{r}_{i}=(x, y, z)_{i}$ is the vector representation of $3 \mathrm{D}$ points, while $\vec{r}$ is all scatter points' geometric center point (first moment, the average values of $\mathrm{x}, \mathrm{y}$, and $\mathrm{z}$, respectively); the summation of second-order distance from all scatters to the center point is considered as clustering (second moment). Besides, Num is total number of datasets, and $i$ means analyzing all 16 datasets in an ergodic system. According to the two new indexes of one model and overall P-values representing the mean confidence level of the model in different datasets, we drew each model in two-dimensional linearity-clustering phase diagram with confidence level color bar. This linearityclustering phase diagram with integrated P-values, called as LCP, can assist us with an all-round analysis of one model's performance. Moreover, it can guide in the discovery of novel and effective models.

\section{Gene Ontology Enrichment}

The genes included in each prognostic model may imply some clear or potential mechanism. Based on this consideration, gene function enrichment was analyzed using the online tool Metascape (Tripathi et al., 2015). This tool is utilized to explore the biological process (BP) and molecular function (MF) of risk genes. Fisher's test was used to estimate significant enrichment. Gene enrichment visualization was done using ggplot2 package of R (Wickham, 2015).

\section{Novel Model Construction}

Using the LCP method, 13 models can be ranked. Among them, the top three models (rank three on the top of the axis) are selected and combined to get a novel model. And the novel model was validated among 16 independent datasets. For clarity, the workflow of the complete analysis process is shown in Figure 1.

\section{Statistic Analysis}

In this study, $\mathrm{P}$-value $\leq 0.05$ was considered to be significant for log-rank test. The value of AUC and C-index $>0.6$ was considered as good performance. $\mathrm{HR}$, and $95 \%$ confidence interval (CI) were calculated to identify low-risk $(\mathrm{HR}<1)$ or high-risk gene signature model $(\mathrm{HR}>1)$. Kaplan-Meier curve was employed to estimate the differences between the high- and low-risk patients.

\section{RESULTS}

\section{Characteristics of Collected Gene Signature Models and OvCa Sample Sets}

By searching and screening the literature, 13 prognosis gene signature models were included in this study; all of them were obtained from Cox regression method. The publications are listed in Table 1. Each study provided its own gene signature, with corresponding coefficients $(\beta)$ or HRs. However, the 13 models showed nearly no overlap for any two models (Supplementary Table S1). By calculating the Jaccard indexes of pairwise models, the overlap of the vast majority of gene signature models was found to be less than 5\% (Figure 2A). The result of GO enrichment showed that various gene signature models were enriched in different $\mathrm{GO}$ terms (Figures 2B, C).

The number of sample sets obtained after filtering is presented in Table 1. Overall, many studies focused on late-stage (III, IV) and high-grade $(2,3)$ OvCa. Notably, of these gene signature models, there were three publications that integrated data from early-stage (I, II, or II) and late-stage (III, IV) OvCa.

\section{Evaluation of Each Model Using 16 Independent Datasets}

The NPI of each gene signature was calculated to label high or low risk for patients in each model. In order to evaluate the performance of a model sufficiently, we analyzed the model's three indicators (HR, C-index, and AUC) simultaneously and obtained the corresponding P-value of log-rank from significance of OS between high-risk and low-risk cohorts. Boxplots were employed to show the actual distribution and distinction of the three indicators and $\mathrm{P}$-value. The results showed the indicators (including AUC, C-index, p-value, and HR) represented the difference among different models (Figure 3). It is difficult to estimate the performance of each model by single indicator alone.

\section{Integrated Indicators for Re-Estimated Prognostic Gene Signature in Ovca With LCP}

For investigating the relationship between the indicators, we analyzed their correlation. As presented in Supplementary Figure S1, HR, C-index, and AUC showed strong collinearity. In contrast, the P-value derived from log-rank test showed some negative correlation. As a consequence, the three collinearity indicators could be mapped into three-dimensional space, and we analyzed their linearity and clustering property to evaluate models from a geometric viewpoint (see Method). In this study, a novel geometric estimating method was proposed for assessment, based on which, we drew the two-dimensional linearity-clustering phase diagram of all modals analyzed (Figure 4). For showing an obvious distinction among the models, we obtained the reciprocals of the linearity fitting residual and clustering results, which represent the gathering degree of scatter points of one model in HR-C index-AUC (HCA) space. The model points located in top right reflected the model performing with high consistency and robustness. The color labels show the integrated P-values of one model and indicate the mean confidence level. As shown in Figure 4A, we found that two models (Riester and Mok) showed good clustering and linearity properties. Meanwhile, three models (Willis, Riester, and TCGA) had higher confidence level than the other models. This prompted us to examine whether combining different independent models with good performance into a novel model might improve the performance further. Therefore, two new models, called WRT (Willis+Riester+TCGA, Combination 1, Supplementary Table S2) and RM (Resier+Mok, 


\section{Literature Retrospect \& Reviews}

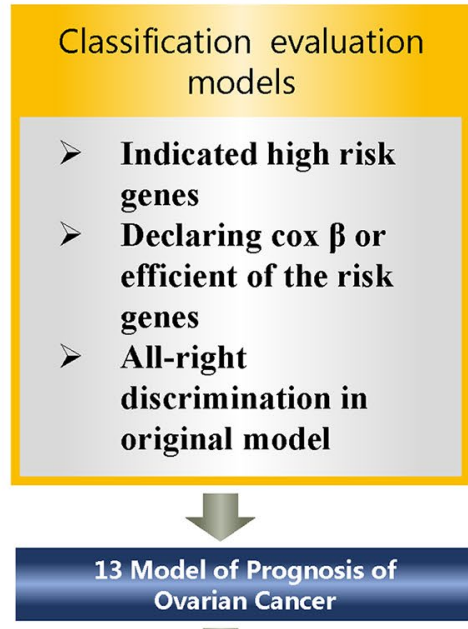

V

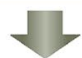
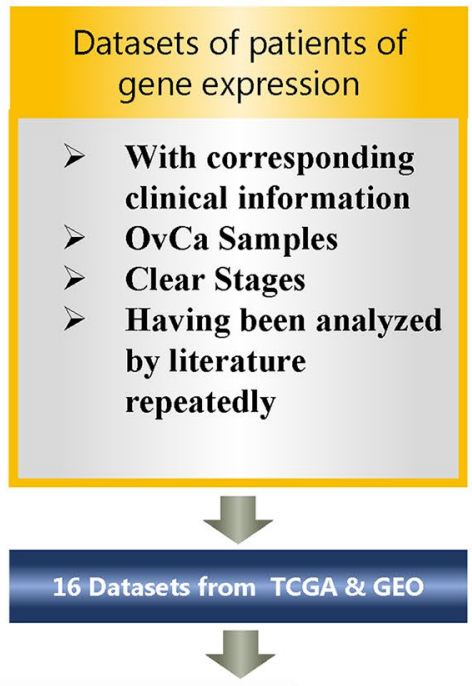

Calculated prognostic index (PI) and corresponding four indicators

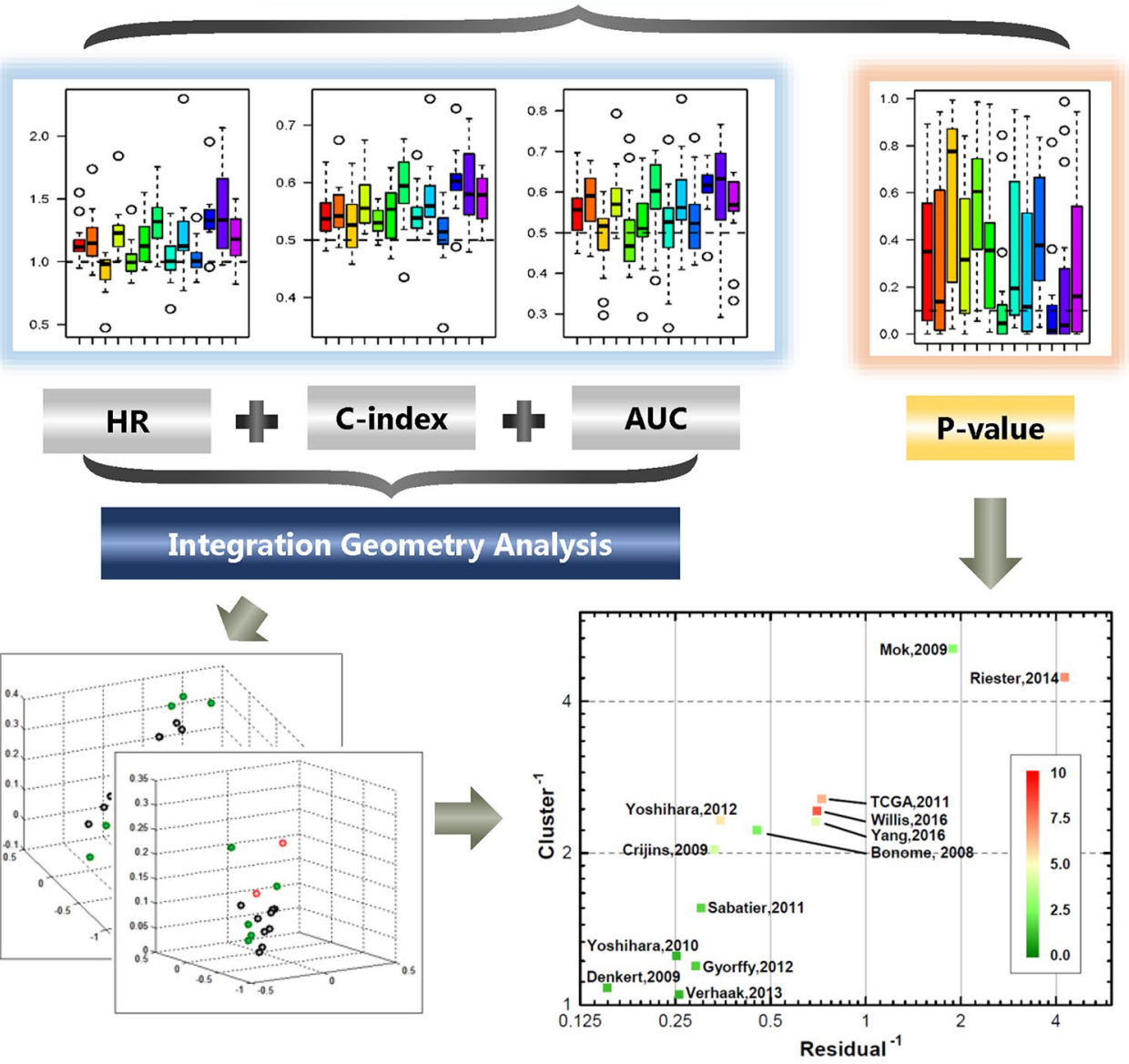

FIGURE 1 | The study flowchart. The chart describes the process of filtering available dataset and method of calculation and analysis. 
TABLE 1 | The 13 published gene signatures for the prognosis of ovarian cancer selected for meta-analysis.

\begin{tabular}{|c|c|c|c|c|c|c|}
\hline Gene signature & $\begin{array}{l}\text { Number } \\
\text { of genes }\end{array}$ & $\begin{array}{l}\text { Number of } \\
\text { samples }\end{array}$ & $\begin{array}{l}\text { Form of } \\
\text { parameter }\end{array}$ & TNM stage & Grade & Dataset \\
\hline Bonome et al., 2008 & 57 & 195 & $\beta$ & III, IV & High grade & GSE26712 \\
\hline Mok et al., 2009 & 200 & 53 & $\mathrm{HR}$ & III, IV & 3 & GSE18520 \\
\hline Denkert et al., 2009 & 300 & 80 & $\beta$ & III, IV & 2,3 & GSE14764 \\
\hline Crijns et al., 2009 & 86 & 110 & $\mathrm{HR}$ & III, IV & $1,2,3$ & GSE13876 \\
\hline Yoshihara et al., 2010 & 88 & 157 & $\beta$ & III, IV & $1,2,3$ & GSE17260, GSE9891 \\
\hline Sabatier et al., 2011 & 7 & 35 & $\mathrm{HR}$ & I, III, IV & $1,2,3, \mathrm{NI}$ & - \\
\hline Network TCGA, 2011 & 193 & 489 & $\beta$ & II, III, IV & $1,2,3$ & TCGA,2011 \\
\hline Yoshihara et al., 2012 & 126 & 300 & $\beta$ & III, IV & 2, 3 & GSE32062 \\
\hline Gyorffy et al., 2012 & 37 & 1287 & $\mathrm{HR}$ & I, II, III, IV & $1,2,3$ & $\begin{array}{l}\text { TCGA, GSE14764, GSE15622, GSE19829, } \\
\text { GSE3149, GSE9891, GSE18520, GSE26712 }\end{array}$ \\
\hline Verhaak et al., 2013 & 100 & 489 & $\beta$ & II, III, IV & High grade & TCGA, GSE9899 \\
\hline Riester et al., 2014 & 200 & 1525 & $\beta$ & I, II, III, IV & High grade & $\begin{array}{l}\text { TCGA, E.MTAB.386, GSE12418, } \\
\text { GSE13876, GSE17260, GSE18520, GSE19829, } \\
\text { GSE26710, GSE30009, GSE32062, GSE9891 }\end{array}$ \\
\hline Willis et al., 2016 & 32 & 1757 & $\mathrm{HR}$ & III, IV & High grade & $\begin{array}{l}\text { TCGA, GSE14764, GSE15622, GSE19829, } \\
\text { GSE3149, GSE9891, GSE18520, GSE26712 }\end{array}$ \\
\hline Yang et al., 2016 & 19 & 484 & HR and $\beta$ & I, II, III, IV & $1,2,3$ & TCGA, GSE9899 \\
\hline
\end{tabular}

combination 2, Supplementary Table S3), were obtained and verified (Figure 4B). The results showed that both WRT and RM models had good performance in the HCA space; they both appeared in the upper right position. Although RM model showed higher position than WRT, it had lower confidence level with green color. On account of this outcome, only WRT was considered as the more accurate model that can satisfy all estimation conditions. The list of genes in WRT is listed in Supplementary Table S4.

\section{Validation Results From Geometric Methods by Clustering Each Single Indicator}

These four indicators (HR, AUC, C-index, and p-value) were individually clustered by unsupervised hierarchical clustering in $\mathrm{R}$ software using the package "pheatmap" (Figures 5A-D). As shown in Figure 5, the different indicators always clustered into two groups - those that performed well or did not. Surprisingly, we always found three models, namely, Willis, TCGA, and Riester, included in the well-performing groups, irrespective of the indicators (Figure 5E).

\section{Gene Ontology Enrichment}

The pathway of genes in the novel WRT combination model was analyzed for GO enrichment (Figures 6A, B, C), and results showed that the risky genes were mainly involved in viral transcription and viral gene expression and were associated with viral infection.

\section{DISCUSSION}

In this study, we evaluated 13 gene signature models from 16 datasets between 2005 and 2017. Although each prognostic model was derived from Cox regression method, some models were computed through a single dataset, while others were obtained through meta-analysis of integrated datasets. Meta-analysis has the advantage of stably merging many studies-for example, the studies by Riester et al. (2014) and Willis et al. (2016) employed metaanalysis to obtain prognostic gene signature. Although the quality of research from different regions and laboratories are very different, some studies have found that there was no significant difference in the study of genetic biomarkers between different regions (Tian et al., 2017). Importantly, some high-throughput experiments also derived good gene signatures by high-quality datasets and advanced algorithms. For example, the studies by Yoshihara et al. (2012) and Yang et al. (2016) relied on high-quality data to obtain good gene signatures. Moreover, we found that more accurate gene signature and stability results were mainly obtained from larger sample size and advanced algorithm application to some degree (Table 1 and Figure 5). In 2014, Waldron et al. and Riester et al. extensively evaluated various gene signature models from previous publications for predicting outcome of patients with OvCa. In 2016, Willis et al. and Yang et al. proposed two new gene signature models for predicting prognosis of OvCa by different methods. Noticeably, there is little intersection between the two gene signatures (Figure 2A). Willis et al. employed meta-analysis and then verified the results in many datasets, while Yang et al. only used the TCGA dataset to train a gene signature model by elastic regulation network. From the results of clustering all gene signatures, there were five models that showed good universality. Generally, the accuracy of predictor estimation for cancer is effected by numerous practical factors, such as methodological heterogeneity, clinical heterogeneity, small sample sizes, batch effects, and lack of independent dataset for validation (Simon, 2005; Baggerly et al., 2008; Dobbin et al., 2008; Sabatier et al., 2009; Koscielny, 2010; Leek et al., 2010; Medicine, 2012). This prompted us to investigate 13 gene signature models across large gene expression datasets.

Based on geometrical concepts, our novel method, proposed in this study, integrated three indicators to estimate prognostic models. As a result, we could not only estimate each model quantitatively and give each model a position intuitively but 
A

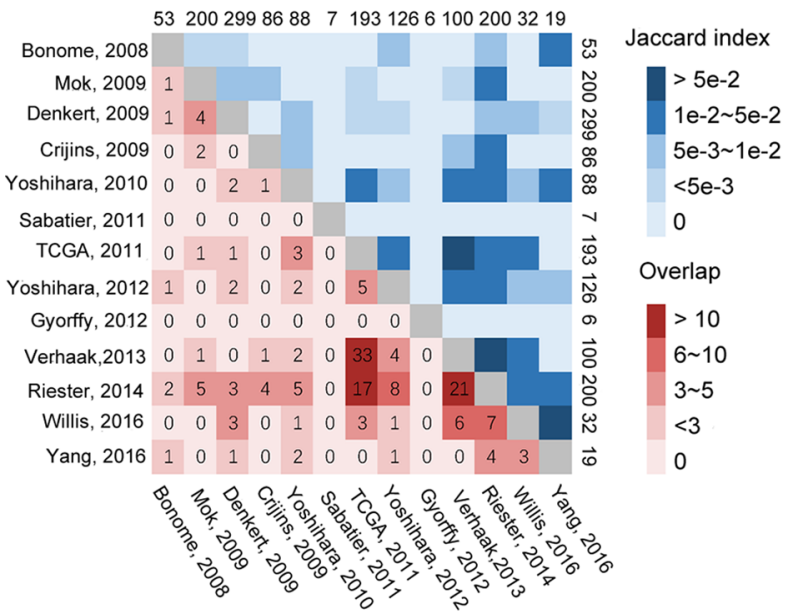

C
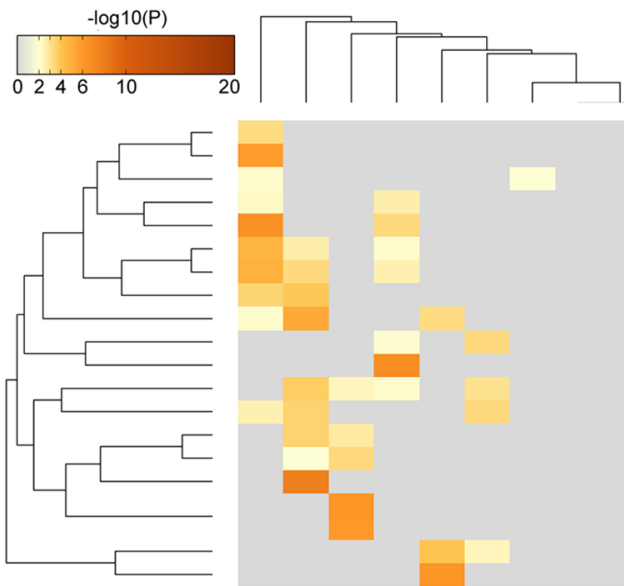

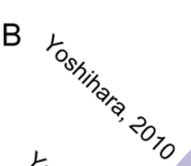

Yostingara, 2012 Bonome, 2008
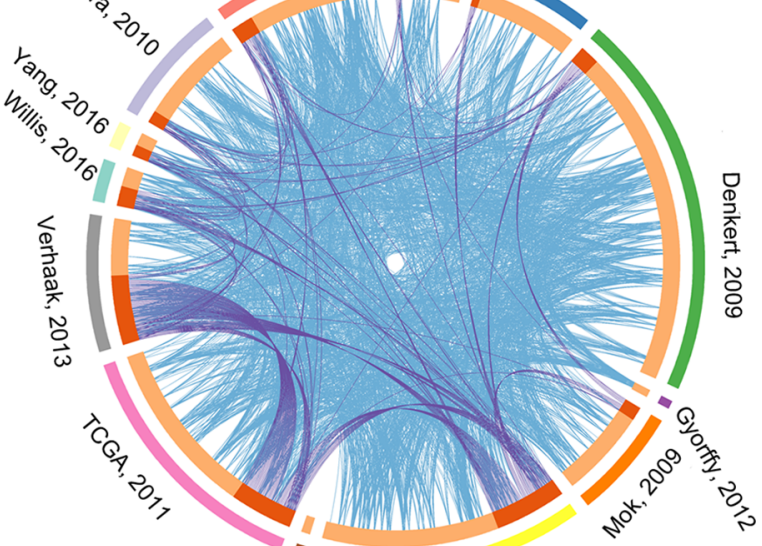

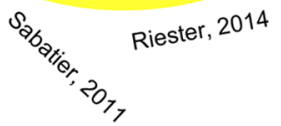

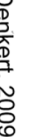

M207: PID RETINOIC ACID PATHWAY

GO:0009205: purine ribonucleoside triphosphate metabolic process GO:0097191: extrinsic apoptotic signaling pathway GO:1901361: organic cyclic compound catabolic process GO:0042110: T cell activation GO:0060326: cell chemotaxis GO:0030155: regulation of cell adhesion GO:0030155: regulation of cell adhesion GO:0030855: epithelial cell differentiation GO:0008285: negative regulation of cell proliferation M7955: SIG INSULIN RECEPTOR PATHWAY IN
hsa05322: Systemic lupus erythematosus hsa05322: Systemic lupus erythematosus
GO:0001568: blood vessel development GO:0044283: small molecule biosynthetic process GO:0061448: connective tissue development GO:0000122: negative regulation of transcription by RNA polymerase II M5884: NABA CORE MATRISOME GO:0060349: bone morphogenesis GO:0042445: hormone metabolic process GO:0042445: hormone metabol hsa05200: Pathways in canc
M167: PID AP1 PATHWAY

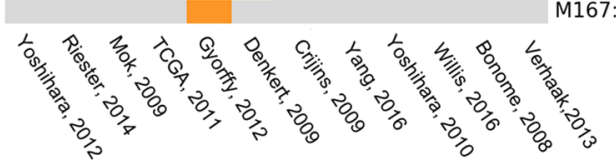

FIGURE 2 | Overlapping of 13 gene signature models that were tested by Jaccard index. The numbers on edges represent the number of genes in each model. (A) The numbers in lattices represent the number of genes overlapping between different models. (B) The relationship of function among the 13 gene signature models. The blue lines represent the connection between two gene signature models. The purple lines represent at least three models associated with each other. (C) The overlapping of GO enrichment and KEGG among 13 gene signature models.

also selected good models to combine into a new model. The results showed that the combination model (WRT model) could perform better than the individual models across the 16 independent datasets. The RM combination model, although good, did not perform as well as the WRT model.

For further explaining the role of WRT model in OvCa, we applied GO enrichment for assaying the genes in the WRT model. We found that risky genes were mainly involved in viral transcription and viral gene expression processes, while protective genes were mainly involved in immune-related processes. Both the biological function and computational results showed that the WRT model exhibited pathways associated with viral infection. Although some cancers are caused by viral infections, the relationship between $\mathrm{OvCa}$ and viruses is unclear. However, some researchers have reported that patients with OvCa in Indian population are infected with human papillomavirus (Shanmughapriya et al., 2012). Other researchers have found a relationship between Chlamydia and the risk of ovarian cancer (Trabert et al., 2018).

\section{CONCLUSIONS}

In summary, our work provides a platform for further investigating the causes of different gene signatures for effective OvCa prognosis. We not only provide a method 
A

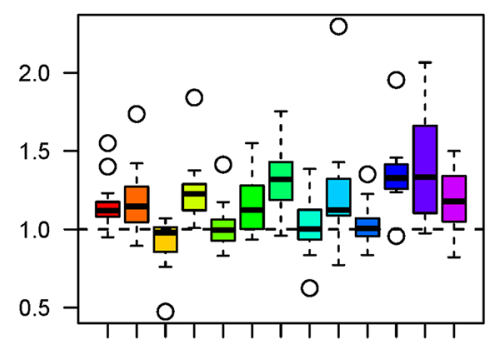

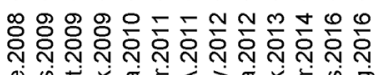

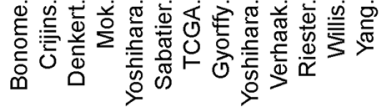

C

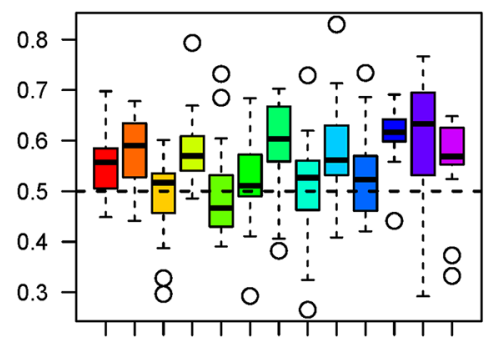

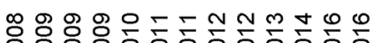

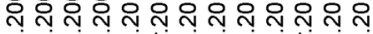

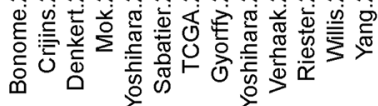

B

C-index

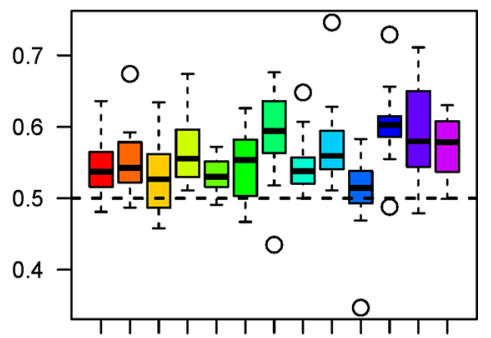

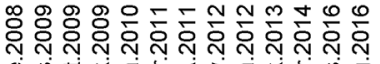

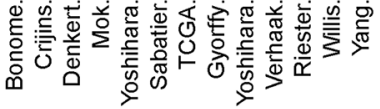

D

p-value of log-rank

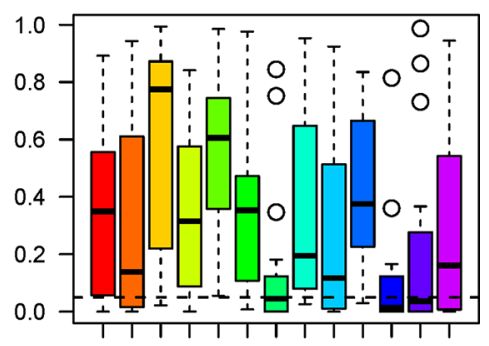

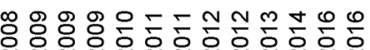

ס

FIGURE 3 | The boxplots of the three indicators and p-value. The three indicators and p-value, HR (A), C-index (B), AUC (C), and P-value (D) of each model of the 16 datasets are depicted by boxplot, respectively. The dashed line represents the threshold for each indicator (HR $=1$ as the threshold, C-index $=0.5$ as threshold, $A \cup C=0.5$ as threshold, and P-value $=0.05$ as significant threshold). The P-values of log-rank (D) from comparison of high-risk and low-risk cohorts are also considered.

\section{A}

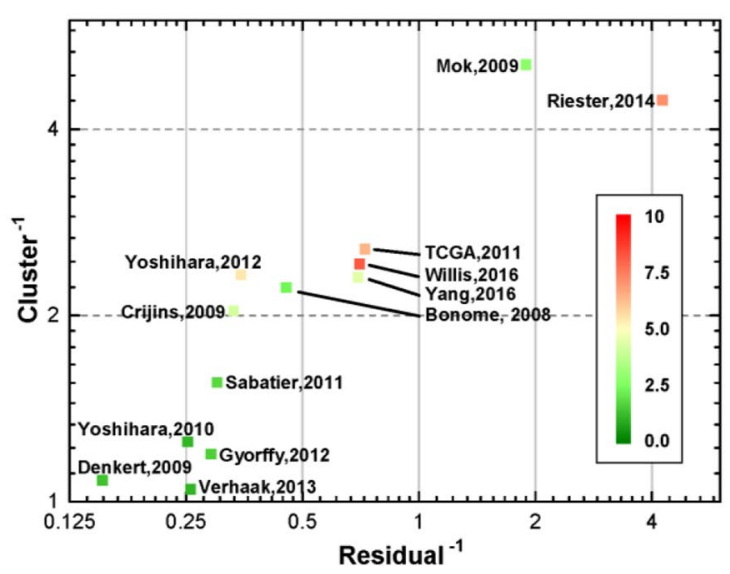

B

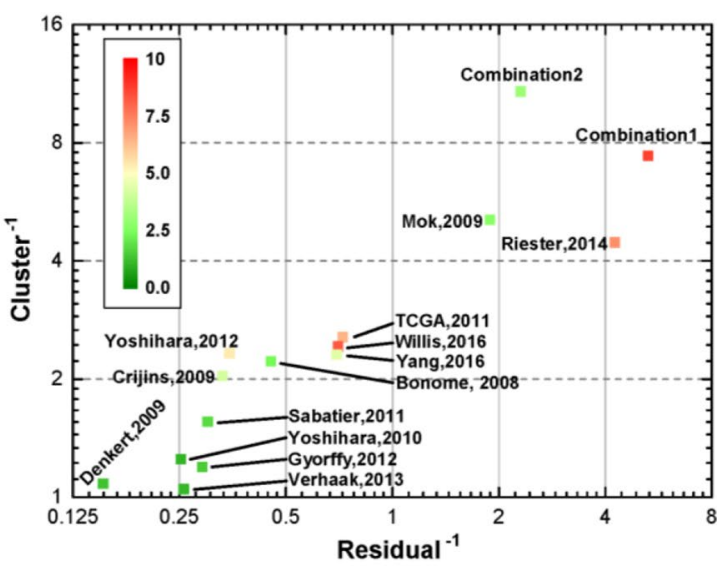

FIGURE 4 | Two-dimensional linearity-clustering phase diagram of models. Tags in the figure denote the various models as described in Table 1. The coordinates show the reciprocals of residual of a line fitting and gathering degree of scatter points of one model in 3D space repetitively. The color of point reflects the overall confidence level. (A) Distribution of the 13 gene signature models in linearity-clustering phase diagram. (B) Distribution of the combination models in linearity-clustering phase diagram. 


\section{A}

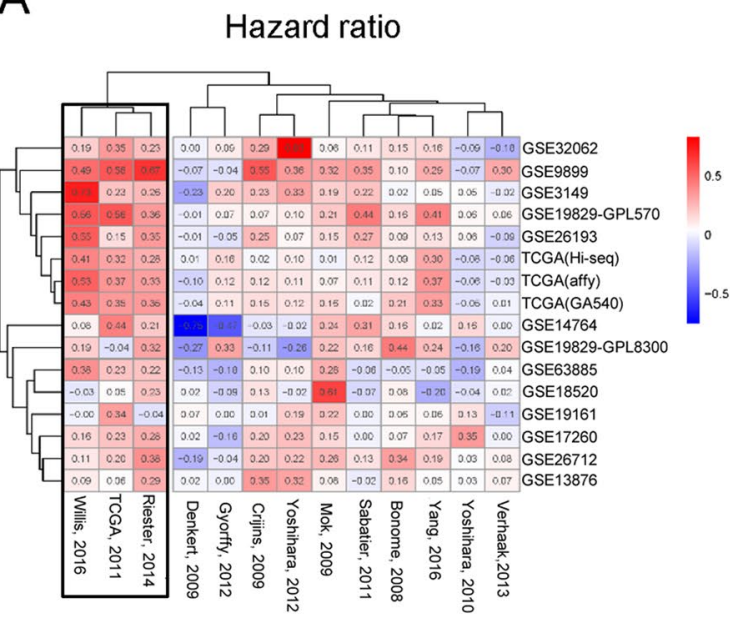

C

$A \cup C$

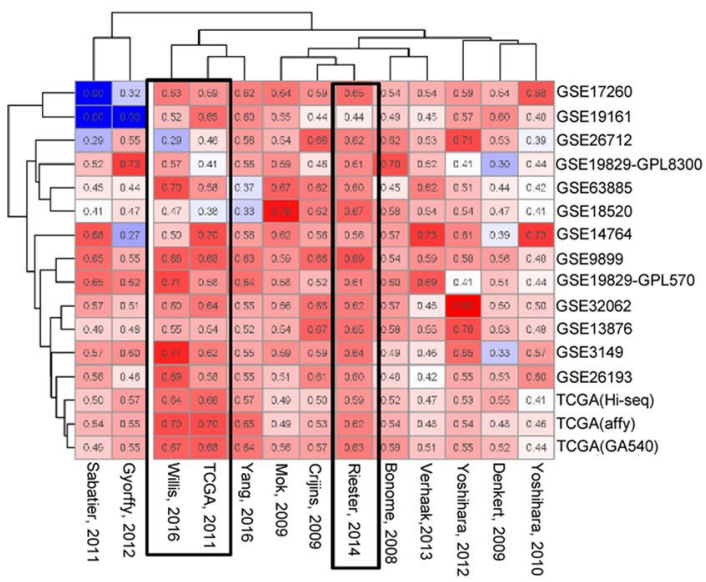

B C-index

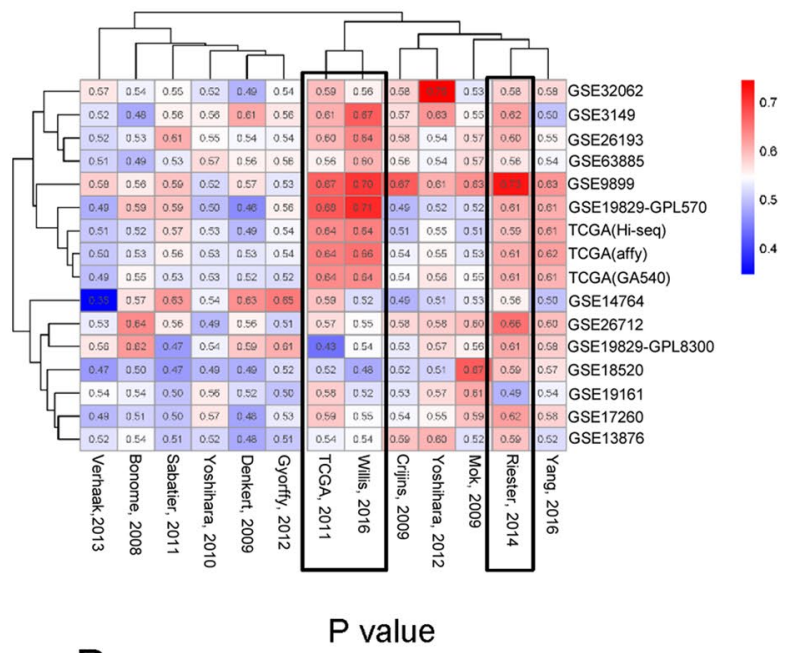

D

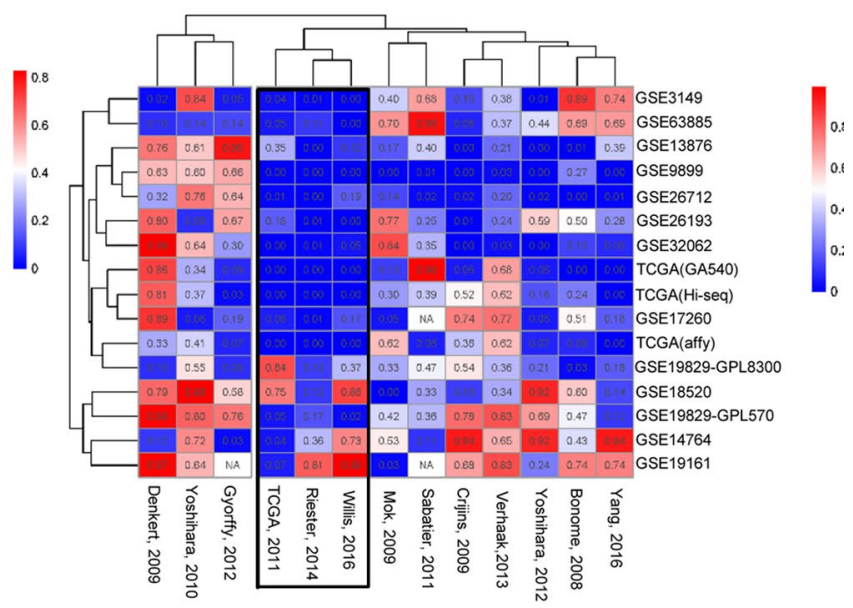

E 3 models (Willis, TCGA, Riester)

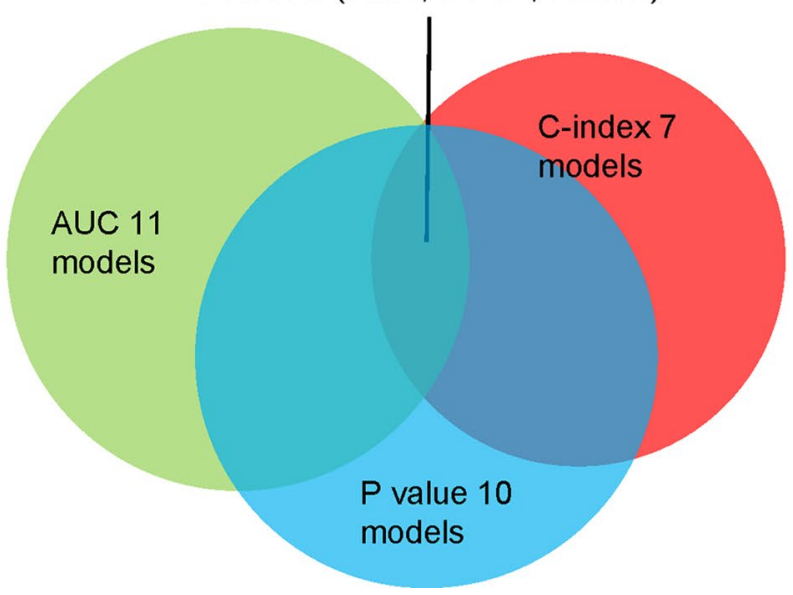

FIGURE 5 | Three indicators clustered across 16 different datasets: (A) HR, (B) C-index, (C) AUC of 5 years. (D) Log-rank p-value of the 13 models clustered in 16 different datasets. (E) The overlapping of the four indicators. 
A

Biological process

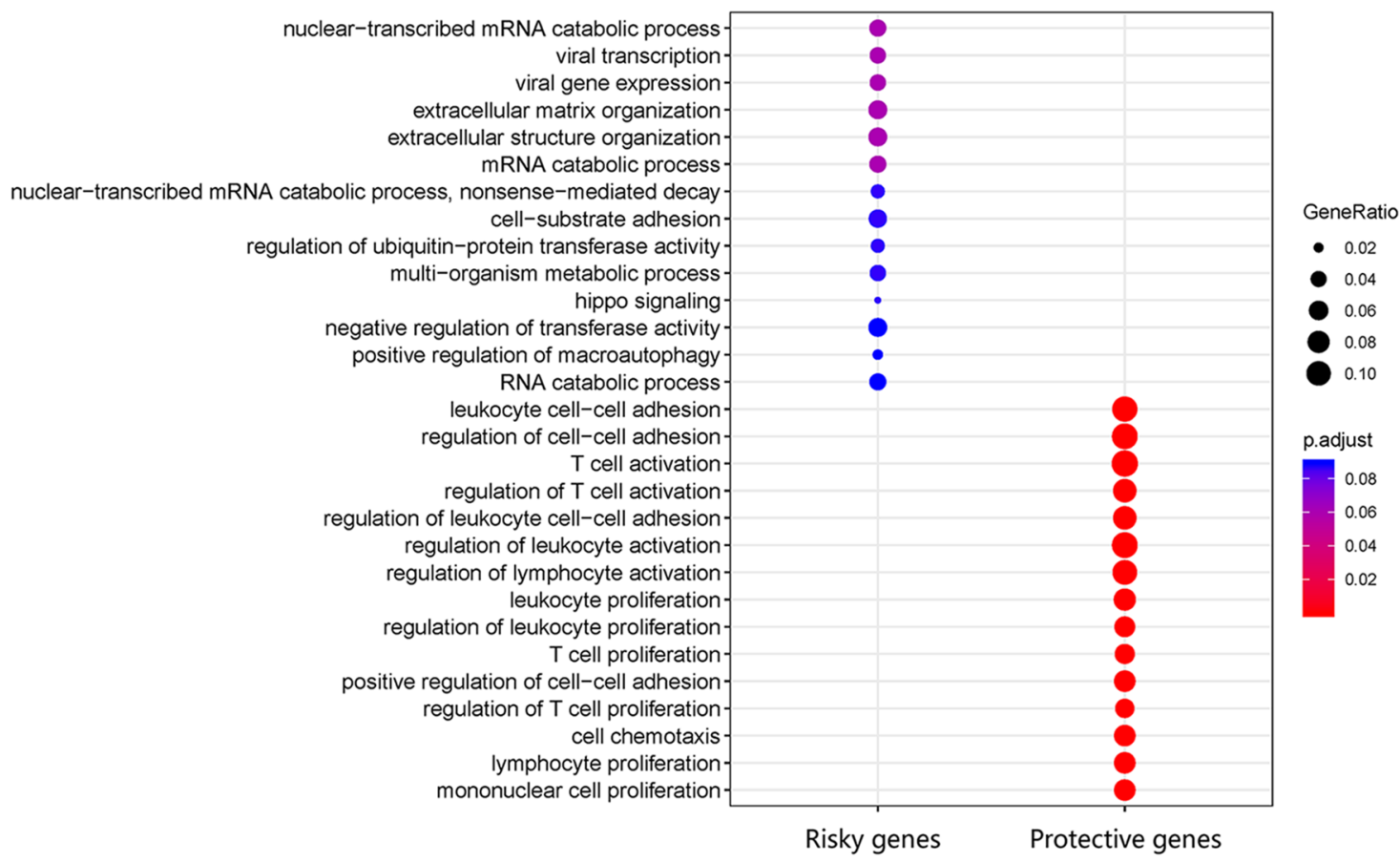

B

Cellular component

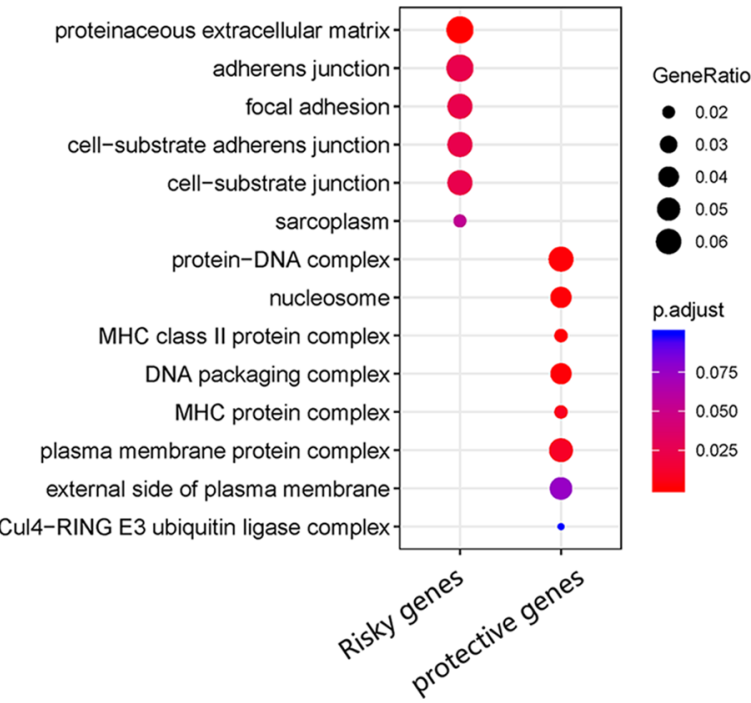

C

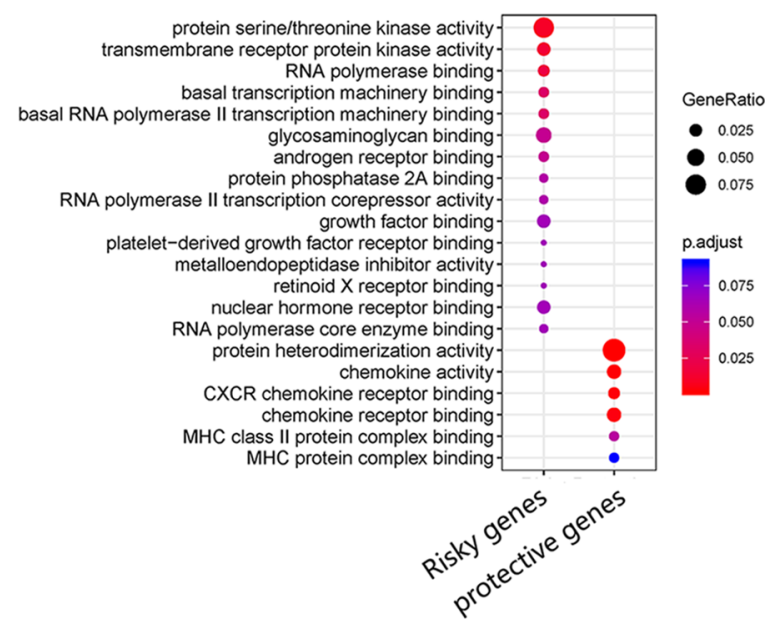

FIGURE 6 | GO enrichment of WRT model. (A) Risky and protective gene enrichment in biological processes. (B) Cellular component and (C) molecular function.

for quantitatively estimating a prognostic model and give each model an intuitive position but also propose a way to obtain a robust model for predicting prognois of OvCa. Noticeably, the integrative model from geometric approach performed better than all original models. Importantly, the multi-platform cross-database combination can obtain more realistic results.

\section{DATA AVAILABILITY STATEMENT}

Publicly available datasets were analyzed in this study. This data can be found here: http://xena.ucsc.edu/welcome-to-ucsc-xena/. TCGA: https://xenabrowser.net/datapages/. GEO: https://www. ncbi.nlm.nih.gov/geo. MetaScape: http://metascape.org/gp/index. html\#/main/stepl. 


\section{AUTHOR CONTRIBUTIONS}

Conceptualization, ZB, YY and JX; Formal analysis, YY; Investigation, $\mathrm{ZB}, \mathrm{JZ}$ and $\mathrm{XS}$; Methodology, JT and $\mathrm{XG}$; Project administration, XS; Software, YY; Supervision, KY; Validation, XL; Visualization, YY and YZ; Writing - original draft, ZB and YY; Writing - review and editing, YZ and KY. All authors read and approved the final version of the manuscript.

\section{ACKNOWLEDGMENTS}

The authors thank the Chinese Academy of Sciences for its computing equipment. We would like to thank Editage (www. editage.cn) for English language editing.

\section{REFERENCES}

Baggerly, K. A., Coombes, K. R., and Neeley, E. S. (2008). Run batch effects potentially compromise the usefulness of genomic signatures for ovarian cancer. J. Clin. Oncol. 26, 1187-1188. doi: 10.1200/JCO.2007.15.1951

Barnes, M., Freudenberg, J., Thompson, S., Aronow, B., and Pavlidis, P. (2005). Experimental comparison and cross-validation of the Affymetrix and Illumina gene expression analysis platforms. Nucleic Acids Res. 33, 5914-5923. doi: 10.1093/nar/gki890

Bentink, S., Haibe-Kains, B., Risch, T., Fan, J. B., Hirsch, M. S., Holton, K., et al. (2012). Angiogenic mRNA and microRNA gene expression signature predicts a novel subtype of serous ovarian cancer. PLoS One 7, e30269. doi: 10.1371/ journal.pone.0030269

Bild, A. H., Yao, G., Chang, J. T., Wang, Q., Potti, A., Chasse, D., et al. (2006). Oncogenic pathway signatures in human cancers as a guide to targeted therapies. Nature 439, 353-357. doi: 10.1038/nature04296

Bonome, T., Lee, J.-Y., Park, D.-C., Radonovich, M., Pise-Masison, C., Brady, J., et al. (2005). Expression profiling of serous low malignant potential, lowgrade, and high-grade tumors of the ovary. Cancer Res. 65, 10602-10612. doi: 10.1158/0008-5472.CAN-05-2240

Bonome, T., Levine, D. A., Shih, J., Randonovich, M., Pise-Masison, C. A., Bogomolniy, F., et al. (2008). A gene signature predicting for survival in suboptimally debulked patients with ovarian cancer. Cancer Res. 68, 54785486. doi: 10.1158/0008-5472.CAN-07-6595

Crijns, A. P. G., Fehrmann, R. S. N., De Jong, S., Gerbens, F., Meersma, G. J., Klip, H. G., et al. (2009). Survival-related profile, pathways, and transcription factors in ovarian cancer. PLoS Med. 6, e1000024. doi: 10.1371/journal. pmed. 1000024

Denkert, C., Budczies, J., Darb-Esfahani, S., Györffy, B., Sehouli, J., Könsgen, D., et al. (2009). A prognostic gene expression index in ovarian cancervalidation across different independent data sets. J. Pathol. 218, 273-280. doi: 10.1002/path.2547

Dobbin, K. K., Zhao, Y., and Simon, R. M. (2008). How large a training set is needed to develop a classifier for microarray data? Clin. Cancer Res. 14, 108114. doi: 10.1158/1078-0432.CCR-07-0443

Ge, L., Tian, J.-H., Li, Y.-N., Pan, J.-X., Li, G., Wei, D., et al. (2018). Association between prospective registration and overall reporting and methodological quality of systematic reviews: a meta-epidemiological study. J. Clin. Epidemiol. 93, 45-55. doi: 10.1016/j.jclinepi.2017.10.012

Gillet, J.-P., Calcagno, A. M., Varma, S., Davidson, B., Bunkholt Elstrand, M., Ganapathi, R., et al. (2012). Multidrug resistance-linked gene signature predicts overall survival of patients with primary ovarian serous carcinoma. Clin. Cancer Res. 18, 3197-3206. doi: 10.1158/1078-0432.CCR-12-0056

Gyorffy, B., Lánczky, A., and Szállási, Z. (2012). Implementing an online tool for genome-wide validation of survival-associated biomarkers in ovarian-cancer using microarray data from 1287 patients. Endocr. Relat. Cancer 19, 197-208. doi: 10.1530/ERC-11-0329

\section{SUPPLEMENTARY MATERIALS}

The Supplementary Material for this article can be found online at: https://www.frontiersin.org/articles/10.3389/fgene.2019.00931/ full\#supplementary-material

FIGURE S1 | Collinearity test of three indicators among 13 gene signature models.

TABLE S1 | The list of genes in the 13 gene signature models.

TABLE S2 | The results of the performance of combination 1 model across 16 independent datasets (WRT).

TABLE S3 | The results of the performance of combination 2 model across 16 independent datasets (RM).

TABLE S4 | Integration model from three great models in OvCa.

Heagerty, P. J., Lumley, T., and Pepe, M. S. (2000). Time-dependent ROC curves for censored survival data and a diagnostic marker. Biometrics 56, 337-344. doi: 10.1111/j.0006-341X.2000.00337.x

Hernandez, L., Hsu, S., Davidson, B., Birrer, M. J., Kohn, E. C., and Annunziata, C. M. (2010). Activation of NF- $\kappa B$ signaling by IKK $\beta$ increases aggressiveness of ovarian cancer. Cancer Res. 70, 4005-4014. doi: 10.1158/0008-5472. CAN-09-3912

Ihaka, R., and Gentleman, R. (1996). R: a language for data analysis and graphics. J. Comput. Graph. Stat. 5, 299-314. doi: 10.2307/1390807

Jemal, A., Siegel, R., Xu, J., and Ward, E. (2010). Cancer statistics, 2010. CA Cancer J. Clin. 60, 277-300. doi: 10.3322/caac.20073

Kang, J., D’andrea, A. D., and Kozono, D. (2012). A DNA repair pathway-focused score for prediction of outcomes in ovarian cancer treated with platinum-based chemotherapy. J. Natl. Cancer Inst. 104, 670-681. doi: 10.1093/jnci/djs177

Kernagis, D. N., Hall, A. H., and Datto, M. B. (2012). Genes with bimodal expression are robust diagnostic targets that define distinct subtypes of epithelial ovarian cancer with different overall survival. J. Mol. Diagn. 14, 214-222. doi: 10.1016/j. jmoldx.2012.01.007

Konstantinopoulos, P. A., Cannistra, S. A., Fountzilas, H., Culhane, A., Pillay, K., Rueda, B., et al. (2011). Integrated analysis of multiple microarray datasets identifies a reproducible survival predictor in ovarian cancer. PLoS One 6, e18202. doi: 10.1371/journal.pone.0018202

Konstantinopoulos, P. A., Spentzos, D., Karlan, B. Y., Taniguchi, T., Fountzilas, E., Francoeur, N., et al. (2010). Gene expression profile of BRCAness that correlates with responsiveness to chemotherapy and with outcome in patients with epithelial ovarian cancer. J. Clin. Oncol. 28, 3555-3561. doi: 10.1200/ JCO.2009.27.5719

Koscielny, S. (2010). Why most gene expression signatures of tumors have not been useful in the clinic. Sci. Transl. Med. 2, 14ps12. doi: 10.1126/ scitranslmed. 3000313

Leek, J. T., Scharpf, R. B., Bravo, H. C., Simcha, D., Langmead, B., Johnson, W. E., et al. (2010). Tackling the widespread and critical impact of batch effects in high-throughput data. Nat. Rev. Genet. 11, 733-739. doi: 10.1038/nrg2825

Li, L., Tian, J., Tian, H., Moher, D., Liang, F., Jiang, T., et al. (2014). Network meta-analyses could be improved by searching more sources and by involving a librarian. J. Clin. Epidemiol. 67, 1001-1007. doi: 10.1016/j. jclinepi.2014.04.003

Lisowska, K., Oibryt, M., Dudaladava, V., Pamuła-Piłat, J., Kujawa, K., Grzybowska, E., et al. (2014). Gene expression analysis in ovarian cancer-faults and hints from DNA microarray study. Front. Oncol. 4, 6. doi: 10.3389/fonc.2014.00006

Mateescu, B., Batista, L., Cardon, M., Gruosso, T., De Feraudy, Y., Mariani, O., et al. (2011). miR-141 and miR-200a act on ovarian tumorigenesis by controlling oxidative stress response. Nat. Med. 17, 1627-1635. doi: 10.1038/nm.2512

Medicine, I. O. (2012). Evolution of translational omics: lessons learned and the path forward. National Academies Press (US). The National Academies Press, Publicity Department, 500 Fifth Street, NW, Washington, DC 20001. doi: $10.17226 / 13297$ 
Mok, S. C., Bonome, T., Vathipadiekal, V., Bell, A., Johnson, M. E., Wong, K. K., et al. (2009). A gene signature predictive for outcome in advanced ovarian cancer identifies a survival factor: microfibril-associated glycoprotein 2. Cancer Cell 16, 521-532. doi: 10.1016/j.ccr.2009.10.018

Network TCGA. (2011). Integrated genomic analyses of ovarian carcinoma. Nature, 2011, 474(7353): 609-615.

Nguyen, L., Cardenas-Goicoechea, S. J., Gordon, P., Curtin, C., Momeni, M., Chuang, L., et al. (2017). Biomarkers for early detection of ovarian cancer. Womens Health 9, 171-185. doi: 10.2217/WHE.13.2

Norris, S. L., Meerpohl, J. J., Akl, E. A., Schünemann, H. J., Gartlehner, G., Chen, Y., et al. (2016). The skills and experience of GRADE methodologists can be assessed with a simple tool. J. Clin. Epidemiol. 79, 150-158.e1. doi: 10.1016/j. jclinepi.2016.07.001

Riester, M., Wei, W., Waldron, L., Culhane, A. C., Trippa, L., Oliva, E., et al. (2014). Risk prediction for late-stage ovarian cancer by meta-analysis of 1525 patient samples. J. Natl. Cancer Instit. 106, dju048. doi: 10.1093/jnci/dju048

Sabatier, R., Finetti, P., Bonensea, J., Jacquemier, J., Adelaide, J., Lambaudie, E., et al. (2011). A seven-gene prognostic model for platinum-treated ovarian carcinomas. Br. J. Cancer 105, 304-311. doi: 10.1038/bjc.2011.219

Sabatier, R., Finetti, P., Cervera, N., Birnbaum, D., and Bertucci, F. (2009). Gene expression profiling and prediction of clinical outcome in ovarian cancer. Crit. Rev. Oncol. Hematol. 72, 98-109. doi: 10.1016/j.critrevonc.2009.01.007

Shanmughapriya, S., Senthilkumar, G., Vinodhini, K., Das, B. C., Vasanthi, N., and Natarajaseenivasan, K. (2012). Viral and bacterial aetiologies of epithelial ovarian cancer. Eur. J. Clin. Microbiol. Infect. Dis. 31, 2311-2317. doi: 10.1007/ s10096-012-1570-5

Siegel, R. L., Miller, K. D., and Jemal, A. (2019). Cancer statistics, 2019. CA Cancer J. Clin. 69, 7-34. doi: 10.3322/caac. 21551

Simon, R. (2005). Development and validation of therapeutically relevant multi-gene biomarker classifiers. J. Natl. Cancer Inst. 97, 866-867. doi: 10.1093/jnci/dji168

Thomson, D. W., and Dinger, M. E. (2016). Endogenous microRNA sponges: evidence and controversy. Nat. Rev. Genet. 17, 272-283. doi: 10.1038/ nrg.2016.20

Tian, J., Zhang, J., Ge, L., Yang, K., and Song, F. (2017). The methodological and reporting quality of systematic reviews from China and the USA are similar. J. Clin. Epidemiol. 85, 50-58. doi: 10.1016/j.jclinepi.2016.12.004

Tothill, R. W., Tinker, A. V., George, J., Brown, R., Fox, S. B., Lade, S., et al. (2008). Novel molecular subtypes of serous and endometrioid ovarian cancer linked to clinical outcome. Clin. Cancer Res. 14, 5198-5208. doi: 10.1158/1078-0432. CCR-08-0196

Trabert, B., Waterboer, T., Coburn, S. B., Brinton, L. A., Sherman, M. E., Lissowska, J., et al. (2018). Serologic markers of infectious agents and ovarian cancer: markers of prior Chlamydia trachomatis infection associated with increased ovarian cancer risk in two independent populations. Cancer Res. 78, 4942. doi: 10.1158/1538-7445.AM2018-4942

Tripathi, S., Pohl, M., Zhou, Y., Rodriguez-Frandsen, A., Wang, G., Stein, D., et al. (2015). Meta- and orthogonal integration of influenza "OMICs" data defines a role for UBR4 in virus budding. Cell Host Microbe 18, 723-735. doi: 10.1016/j. chom.2015.11.002

Verhaak, R. G., Tamayo, P., Yang, J. Y., Hubbard, D., Zhang, H., Creighton, C. J., et al. (2013). Prognostically relevant gene signatures of high-grade serous ovarian carcinoma. J. Clin. Invest. 123, 517-525. doi: 10.1172/JCI65833

Waldron, L., Haibekains, B., Culhane, A. C., Riester, M., Ding, J., Wang, X. V., et al. (2014). Comparative meta-analysis of prognostic gene signatures for late-stage ovarian cancer. J. Natl. Cancer Instit. 106, dju049. doi: 10.1093/ jnci/dju049

Wickham, H. (2015). ggplot2. Wiley Interdiscip. Rev. Comput. Stat. 3, 180-185. doi: 10.1002/wics. 147

Willis, S., Villalobos, V. M., Gevaert, O., Abramovitz, M., Williams, C., Sikic, B. I., et al. (2016). Single gene prognostic biomarkers in ovarian cancer: a metaanalysis. PLoS One 11, e0149183. doi: 10.1371/journal.pone.0149183

Yang, R., Xiong, J., Deng, D., Wang, Y., Liu, H., Jiang, G., et al. (2016). An integrated model of clinical information and gene expression for prediction of survival in ovarian cancer patients. Transl. Res. 172, 84-95.e11. doi: 10.1016/j. trsl.2016.03.001

Yoshihara, K., Tajima, A., Yahata, T., Kodama, S., Fujiwara, H., Suzuki, M., et al. (2010). Gene expression profile for predicting survival in advanced-stage serous ovarian cancer across two independent datasets. PLoS One 5, e9615. doi: 10.1371/journal.pone.0009615

Yoshihara, K., Tsunoda, T., Shigemizu, D., Fujiwara, H., Hatae, M., Fujiwara, H., et al. (2012). High-risk ovarian cancer based on 126-gene expression signature is uniquely characterized by downregulation of antigen presentation pathway. Clin. Cancer Res. 18, 1374-1385. doi: 10.1158/10780432.CCR-11-2725

Conflict of Interest: The authors declare that the research was conducted in the absence of any commercial or financial relationships that could be construed as a potential conflict of interest.

Copyright (c) 2019 Bing, Yao, Xiong, Tian, Guo, Li, Zhang, Shi, Zhang and Yang. This is an open-access article distributed under the terms of the Creative Commons Attribution License (CC BY). The use, distribution or reproduction in other forums is permitted, provided the original author(s) and the copyright owner(s) are credited and that the original publication in this journal is cited, in accordance with accepted academic practice. No use, distribution or reproduction is permitted which does not comply with these terms. 